\title{
Towards Dynamic Financial Valuation of Social Licence to Operate under Uncertainty
}

\author{
$\underline{\text { T. Tarnopolskaya }}^{a}$ and A. Littleboy ${ }^{b}$ \\ ${ }^{a}$ CSIRO, North Ryde, NSW \\ ${ }^{b}$ CSIRO Mineral Resources Flagships, Pullenvale, Qld \\ Email: tanya.tarnopolskaya@,csiro.au
}

\begin{abstract}
The concept of a Social Licence to Operate (SLO), first introduced in the minerals industry, has significantly evolved over the last decade and is currently viewed as a broad, ongoing approval and acceptance of business activities by society and stakeholders. It is gaining prominence outside the minerals industry, in the energy and manufacturing sectors.
\end{abstract}

SLO is broadly recognized as crucial for profitable and sustainable mineral resource extraction operations. Recent research shows that the reduction in project value due to the lack of the SLO can be as high as $70 \%$. This motivates a strong interest from industry to assess the financial value of SLO to the company, in order to prioritise the SLO investments alongside other operational investments and to connect SLO with the project value, business risk and decision-making. However, there are still no accepted methodologies available to achieve this.

The consensus emerging in the literature is that the quality of company engagements with stakeholders/communities is among the most important factors influencing the SLO outcome. However, there are other factors affecting SLO outcome, such as existing and future economic, environmental and political conditions in the region; environmental and social impacts generated by mining operations; the company's ability to anticipate future risks and their proactivity in engagements with stakeholders and communities. The long timeframes for mining operation ( $>30$ years) also mean that technological innovation can drive significant change in the value of economic, environmental and social assets over the life of a mine. Capturing this requires systematic quantitative work with deep qualitative grounding in context. Combining quantitative and qualitative analysis is a major challenge in SLO research.

In this paper, we discuss the possibility of quantitative modeling of SLO and propose several quantitative approaches and a novel integrated conceptual framework for dynamic financial valuation of SLO that accounts for such important SLO features as:

- Complex and non-linear interactions between industrial operations, communities and stakeholders responses, business decision making and company's value;

- Dynamic nature of SLO and its evolution over the lifecycle of mineral operations;

- Multiple uncertainties surrounding mining operations and SLO outcomes;

- Importance of flexibility and timing of business decisions in SLO management;

- Importance of anticipating future risks and proactive engagements with stakeholders/community;

- Unknown response to managerial actions from community and stakeholders.

The proposed framework is based on the integrated real options, adaptive management and risk analysis approaches.

This paper lays the foundation for development of decision support tools in which economic, social and environmental challenges are addressed simultaneously during the lifecycle of the minerals extraction operations, to achieve sustainable operations with robust SLO.

Keywords: Financial valuation, social licence to operate, real options, adaptive management, resilience 


\section{INTRODUCTION}

The concept of a Social Licence to Operate (SLO), first introduced in the minerals industry, has significantly evolved over the last decade and is currently viewed as a broad, ongoing approval and acceptance by the community and other stakeholders of the impact, behavior and the activities of the mining project (e.g. Thompson and Boutilier, 2011; Boutilier et al., 2011; Lacey et al., 2012). It is rapidly gaining prominence outside the minerals industry, in the energy and manufacturing sectors (e.g. Lacey et al., 2014).

It is broadly recognised that SLO is crucial for profitable and sustainable mineral resource extraction operations. Industrial data suggest that the negative impact of lack of SLO on mineral resources operations has significantly grown over the last few years and is now considered as the third largest business risk (Brereton, 2015). The International Council of Mining and Metals (ICMM) recently documented exponential growth in cases of significant company-community conflicts worldwide (ICMM, 2015). It has been demonstrated (Henisz et al., 2013; Henisz, 2015) that lack of SLO could reduce the market value of the mining company by up to $70 \%$.

The importance of financial valuation of SLO has been recognized in the literature (e.g. Jones et al., 2011; Cooper, 2014; Charles, 2015). The criticality of SLO to the viability of an operation suggests a need to find ways of managing it as part of risk management and good governance, with all the rules, norms and corporate governance practices that this implies.

Over the last few years, several new strands of SLO research have emerged, including: (a) measuring and modeling the factors influencing SLO; (b) translation of environmental and social risks into business costs via company-community conflicts; and (c) developing a system-based view of SLO. Practical tools for financial valuation of sustainability investments are being developed (see, e.g., IFC (2012)) and have many features relevant to SLO. However, the research is still limited and there are virtually no quantitative methods for SLO evaluation or accepted methodologies available to assess the financial value of SLO that would reflect important features of SLO. The complex, nonlinear, interactive, rapidly changing and highly uncertain nature of SLO outcomes requires new integrated framework for its valuation.

This paper proposes a novel conceptual framework for dynamic financial valuation of SLO, with a focus on quantitative approaches that reflect the important features of SLO recognized in the research literature, such as its dynamic and interactive nature, complexity and uncertainty. The proposed framework is based on the integrated real options, adaptive management and stakeholder induced risk analysis approaches.

\section{SOCIAL LICENCE: LITERATURE REVIEW}

Comprehensive reviews of SLO related social science literature can be found, e.g., in Zhang and Moffat, 2015, and Prno and Slocombe, 2014. This section briefly reviews the recently emerged strands of SLO research most relevant to formulating a quantitative framework for dynamic valuation of SLO.

\subsection{Measuring and modeling the SLO}

Thompson and Boutiller (2011) proposed a cumulative hierarchical model that describes SLO as a continuous process of social capital development that evolves through four levels: withholding/withdrawal, acceptance, approval and co-ownership. The four levels are separated by the three transition (boundary) criteria, and the process can move in both directions. However, empirical validations of the model have been unsuccessful so far (Boutillier and Thompson, 2011).

Moffat and Zhang (2014) re-examined the manner in which SLO is granted and maintained, and analyzed the critical elements of SLO by conducting a longitudinal study in an Australian mining region. They identified the central role of building trust with local communities and established the relationships between the negative impacts from mining operations, the procedural fairness and the quality of engagement with communities in gaining and maintaining SLO. SLO construction is highlighted in this work as a dynamic process.

Zhang and Moffat (2015) examined how the community's perception of the balance of benefits and negative impacts of mining affects the acceptance of mining activities. Through case studies, they identified that the perceived environmental impact of a resource operation was the factor where little compromise is possible and that often lead to rejection of mining (see similar findings in Frank et al., 2014). Confidence in government institutions was identified as an important factor in moderating the effect of environmental impact on acceptance of mining. The strength of the relationships between different factors and the 
acceptance by the communities was quantified through the application of social psychology and multivariate statistics.

\subsection{Translation of environmental and social risks into business costs}

A comprehensive mechanism of translation of unmitigated perceived environmental and social risks, resulted from mining operations, into business costs via the company-community conflict was proposed by Davies and Frank (2011) and Franks et al. (2014). They identified a broad range of conflicts (from low-level tension to a complete relationship breakdown) and different types of costs, including material damage, lost productivity, lost opportunity, delays, disruptions, security, reputation etc. The failure of companies to respond to stakeholders concerns about risk and the timing of company response to conflict were identified as important factors affecting the business costs. This agrees with findings that proactive and early stakeholder engagement, in response to anticipated risks, is crucial for company's value (Henisz, 2015).

Moffat (2015) found that the reported and underlying causes of social conflicts are usually different, and identified distribution of benefits and poor consultation as underlying causes of conflict. Moffat (2015) and Frank et al. (2014) also observed that company-stakeholders conflicts can be a means through which communities and stakeholders communicate their perception of risks and therefore can influence company's decisions making.

\subsection{System-based view and resilience of SLO}

The complex, interactive, rapidly changing and highly uncertain nature of issues surrounding SLO (such as mineral development, local livelihoods, governance strategies and multiple local and global variables) has been recently recognised in the research literature and lead to development of a system-based conceptual qualitative framework for assessing SLO outcomes in the mining industry (Prno and Slocombe, 2014). The framework is based on complex adaptive systems and resilience theories, and empirical results of a multi-year case studies. It focuses on the identification of key system variables and highlights the importance of context, emergence, uncertainty, evolution, feedback, multiple stable states and thresholds.

The system-based approach introduces the notion of a resilient SLO. Prno and Slocombe (2014) define SLO resilience as a widespread community approval which is maintained throughout a mine's lifecycle, despite crisis events and stresses on company-community relationship. Multiple empirical evidence support the existence of SLO resilience through the role of the 'reputational capital' in reducing future risk and speeding up recovery from disruptions (see Dorobantu et al., 2013; Henisz, 2015; references in Prno and Slocombe, 2014). Despite significant challenges and lack of quantitative models, Prno and Slocombe (2014) demonstrated the viability of complex adaptive systems and resilience theories for assessing SLO.

\section{EXISTING FINANCIAL VALUATION TOOLS FOR SUSTAINABILITY INVESTMENTS}

Financial valuation tools for aspects of social licence are emerging. Tools reported in the literature are: the Financial Valuation Tool (FV Tool) for Sustainability Investment (IFC, 2012); and SKM Social Licence Valuation Framework (SLVF) (Cooper, 2014). Both argue a strong business case for financial valuation of SLO and it is likely that individual companies may have their own bespoke versions as part of their risk governance. The focus of these financial valuation tools is on identification of risks and flow-on effects from community opposition or conflict to the project, and the financial costs and benefits of developing effective community investment solutions versus 'doing nothing' approach. The FV Tool calculates the net present value return to the company from the portfolio of sustainability investments, including value protected (through risk mitigation) and value created (through productivity gains and cash savings). Main components of the FV Tool include: stakeholder analysis; traditional NPV analysis of two scenarios: base case (basic compliance with regulations) and a case with additional community investments; risk quantification and analysis; value protection through risk mitigation; value creation through community investments; assessment of quality of sustainability investment.

Despite the promising start, the existing financial valuation tools are essentially static and passive, and do not account for multiple uncertainties affecting SLO outcomes and a need for flexibility in business decisions in the face of uncertainty. A valuation tool that can capture lead indicators of conflict and the systematic social investments required to address them, in order to prevent conflict arising, is the subject of much discussion. The social science literature outlined in the previous section suggests that the development of such a predictive valuation tool requires new thinking about the complex, dynamic, highly interactive and uncertain nature of the relationship between resource operation and community. This will be a subject of the following section. 


\section{A PROPOSED FRAMEWORK FOR DYNAMIC FINANCIAL VALUATION OF SLO}

A simplified conceptual model of SLO outcome formation and its effect on company's value is presented in Fig. 1, based on the research reported in the literature and discussed in the previous section. The mechanism includes the following main causal relationships:

- Existing and uncertain future economic, social and environmental conditions in the region affect the impacts of mining operations on environment, society and economy during the project life time.

- The actual mining impacts translate into the perceived impacts through the eyes of stakeholders/communities.

- Environmental initiatives and community investments by the company (SLO investments) affect the SLO outcome.

- The balance of the perceived impacts/benefits, amplified or diminished by the quality of engagements with community/stakeholders and other factors (such as governance) determines the attitude of the communities/stakeholders towards the mining.

- Unmitigated environmental and social risks motivate community-company conflicts that result in business costs and loss of company's value.

- Community and stakeholders support provides a buffer for negative influences, thus affecting the company value.

- The company-stakeholder conflicts and their costs to companies, in turn, influence companies' decisionmaking process and the way they address environmental and social risks.

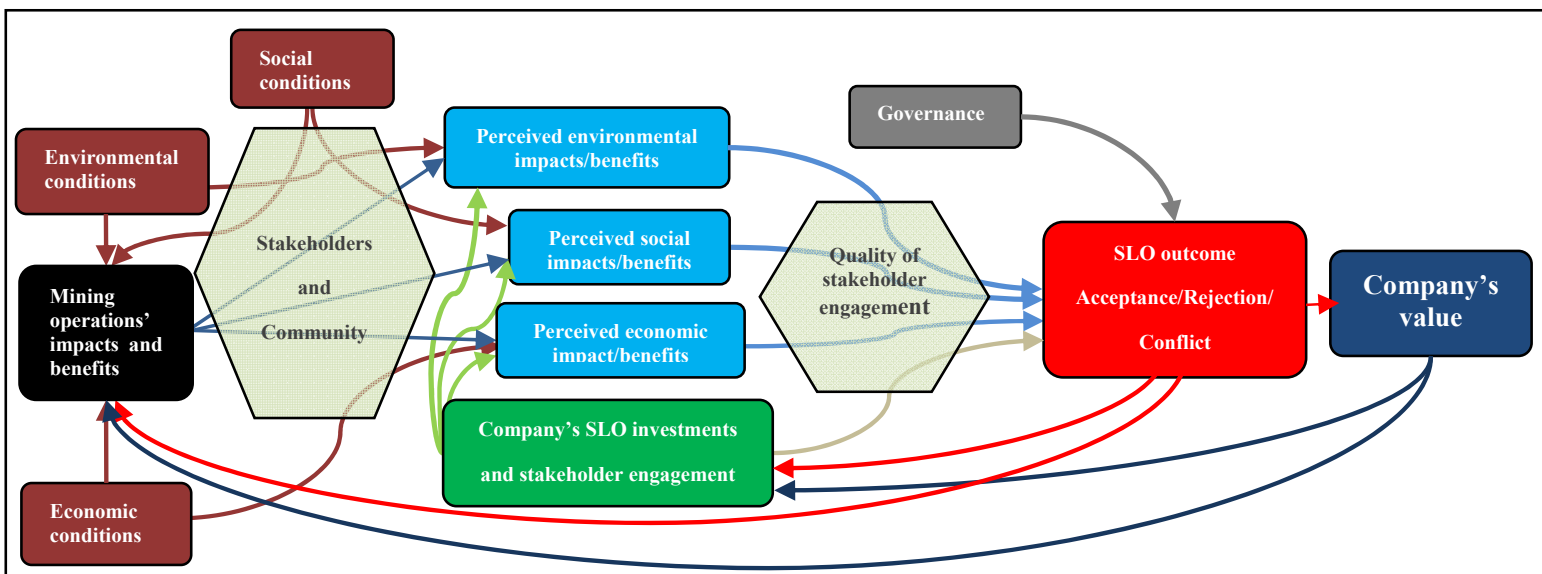

Figure 1. Conceptual model of social licence and its effect on company's value.

The following sections discuss the quantitative approaches capable to reflect the above mechanism. Because of the page limit, we omit the important issue of qualitative analysis of community investments and stakeholder engagement, and refer the readers to the FV Tool (IFC, 2012) where it is adequately reflected.

\subsection{Stakeholder Engagement as a Real Option: Value of Flexibility and Timing}

A dynamic and highly uncertain nature of the relationship between resource operation and community requires a dynamic approach for financial valuation of SLO. Real options analysis, a dynamic valuation and optimal decision under uncertainty methodology with emphasis on value of flexibility and optimal timing of business decisions under uncertainty, is well suited to accommodate such features.

According to the real options concept, flexibility to adapt to changing circumstances is crucial for successful business operation under uncertainty. Optimal flexible management in the face of uncertainty adds value to the project. We suggest that community investments and stakeholder engagements by the company can be viewed as additional flexibility (real option), alongside the traditional operational and strategic flexibilities, that company can utilize to increase the project value if they are implemented in an optimal manner at optimal time, in response to the resolution of uncertainty. In light of ROV concept, the value of SLO is the difference of company's value with optimal flexible community investment under uncertainty, and the value of 'doing nothing'. The value of the risk of conflicts due to perceived mining impacts can be incorporated into the company value without community investments. The difference with the existing financial valuation 
tools (FV Tool and SLFV) is that in the proposed approach the value created will be computed through the dynamic optimization of sequential business decisions under uncertain economic, social and ecological environment. Such approach would allow anticipating future risks and proactive risk mitigation.

The main challenges in using the real options approach for SLO investment decisions are: the lack of a model for community acceptance of mining operations as a stochastic index; and a lack of understanding of the community/stakeholder responses to managerial actions. This calls for approaches developed in the Adaptive Management field (see Section 4.2). Identifying and modeling key uncertainties affecting the minecommunity relationship and their effect on environmental impacts and social benefits from mining is an important starting point of the analysis (see Sections 4.3 and 4.4). Assessment of the quality of sustainability investments alongside their costs is essential for optimizing the investment decisions, and the experience accumulated in FV Tool (2012) will be very useful.

\subsection{Adaptive Management of SLO Outcome: Learning by Doing}

Uncertain and poorly understood community/stakeholders responses to managerial actions are major challenges for financial valuation of SLO. Adaptive management, or 'learning by doing', was specifically developed to handle decision problems where the model of the system behavior is unknown and presents a promising way to deal with such issues. Adaptive management solutions optimize action (assuming that the current knowledge of the system might not be correct) to maximize the chance of achieving the objective (in our case - achieving SLO) by explicitly accounting for future learning opportunities. An approach to modeling the evolution of community acceptance is suggested in Section 4.6.

Both real options and adaptive management approaches are sequential decisions under uncertainty methodologies that promote flexible decision making that can be adjusted in the face of uncertainties. However, existing adaptive management methodologies are not suitable for problems with multiple uncertainties and non-stationary stochastic behavior of variables inherent in the SLO dynamics. Adapting advanced real options techniques to use in the adaptive management is one of the research challenges.

Resilience is an offshoot of complex adaptive system approach which is characterised by multiple equilibriums. Once the predictive models for mine-stakeholders interaction are developed, the analysis of SLO resilience will be part of the real options/adaptive management solution. However, development of realistic quantitative models for components of SLO system presents a major challenge.

\subsection{Modelling the Impacts and Benefits from Mining Operations}

Understanding the environmental and social impacts of mining operations over the mine life cycle is crucial in order to anticipate the environmental and social risks of mining operations. The mining impacts and benefits are significantly affected by multiple uncertainties surrounding the minerals operations, including market, geological, political, climate and other uncertainties. The life cycle analysis of the mining project, with the account of water usage and environmental impacts, combined with the forecasting and Monte Carlo simulation of the risk factors, can provide a basis for the analysis. In addition, an identification of the stakeholders and modelling the distribution of stakeholders' benefits over the mine life cycle under uncertainty is needed to identify potential social risks.

\subsection{Perceived Impacts and Benefits by Community/Stakeholders}

Prospect theory (Kahneman \& Tverski, 1979) suggests that humans perceive gains and losses in different ways. A key characteristic of human attitude to changes is that "losses loom larger than gains". According to the prospect theory, the perceived value is a function of two arguments: (1) the reference point (the initial conditions); and (2) the deviation from the reference point. The marginal value of both gains and losses decreases with their magnitude (a so-called 'diminishing value' effect). This functional form provides a basis for the analysis of the perceived impact and benefits of mining by community and stakeholders, and is consistent with the evidence that it is difficult for the company to offset the negative environmental impacts of the mining operations by producing positive impacts through the social investments (Zhang and Moffat, 2015). The value function needs to be calibrated through the systematic surveys of community/stakeholders over the mine life time.

\subsection{Identification, measurement and modeling the stakeholder-induced risks}

Risk quantification is a central part of the FV Tool (IFC, 2012; Jones, 2012) which identified, through multiple case studies, several common risk types and analyses them using traditional operational risk 
methods. Although we do not discuss the tool here, interested readers can refer to the above documents. One of the difficulties in analysing the stakeholder-induced risk is that companies generally do not aggregate the stakeholder-relates losses, and lack of data presents a major challenge. Nevertheless, case studies in IFC, 2012, demonstrated that a considerable body of knowledge already exists in the industry with respect to linking the environmental impacts of mining operations with the expected losses from the potential companycommunity conflicts (see, e.g., Cooper, 2014; Jones et al, 2011, Franks et al, 2014).

\subsection{Forecasting and Monitoring Dynamics of Community Acceptance of Mining (SLO Outcome)}

As discussed in Section 4.1, predictive models of evolution of community acceptance of mining are essential for development of a dynamic SLO valuation framework. Development of such models would require a comprehensive understanding of community composition, aspirations, key actors and power structures, which is specific for each case study, and its evolution as a mine advances through its life cycle. There is still lack of understanding of how SLO is granted and how the competing stakeholder views should be addressed. A considerable effort is required to clarify these issues.

Dynamic models for SLO evolution can be developed through the construction of a detailed value tree that simultaneously includes all relevant impacts and benefits affecting each stakeholder group, with importance weights derived using regression models (Sparks, 2015). A real-time forecasting and monitoring system that assesses performances over time can be developed by collecting data in a systematic and continuous way throughout life cycle of the mine. The forecasts can be used to assess whether the acceptance/rejection of the mine changes and a monitoring system in place will identify when the value is changing faster than expected and the monitoring strategy can be used to identify unusual trends and adjust the models. Identification of the

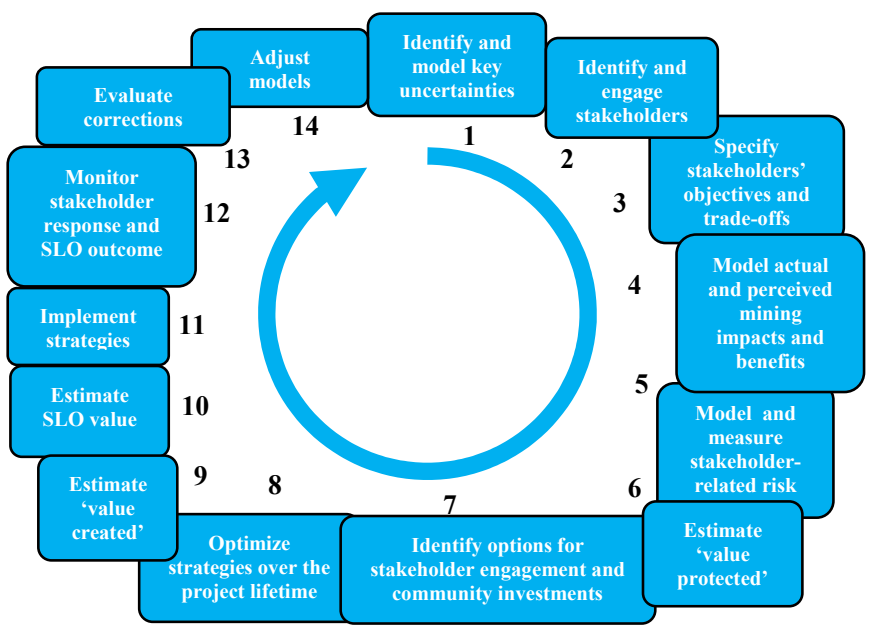

Figure 2. One iterative cycle of dynamic valuation and adaptive management of SLO. thresholds and tipping points that trigger change between the SLO regimes is expected as part of the analysis.

\subsection{Summary of Dynamic Valuation Framework}

Figure 2 shows one iterative cycle of dynamic valuation of SLO, based on the integrated real options, adaptive management and risk analysis framework. Systematic monitoring of stakeholder responses to managerial actions, evaluating SLO outcome and adjusting the models accordingly are crucial steps of the valuation framework.

\section{DISCUSSION AND CONCLUSIONS}

We proposed a novel conceptual quantitative framework for dynamic

financial valuation of SLO that reflects complex, dynamic, highly interactive and uncertain nature of the relationship between resource operation and community. The proposed framework is based on the integrated real options, adaptive management and risk analysis approaches and lays the foundation for development of decision support tools in which economic, social and environmental challenges are addressed simultaneously during the lifecycle of the minerals extraction operations, to achieve sustainable operations with robust SLO.

We identified many challenges and research gaps on the way to developing a comprehensive predictive dynamic decision analysis and financial valuation of SLO. Development of a better understanding and models of the components of SLO system would present a useful starting point for an integrated framework to value the 'social' and hence prioritise investment decisions. Required first steps include: development of a conceptual model for how SLO is granted; a real-time forecasting and monitoring system for dynamic analysis of stakeholder/community acceptance of mining; analysis of mining impacts and distribution of social benefits from mining under multiple uncertainties; development of a combined real options/adaptive management framework for SLO analysis based on the experience from socio ecological systems modeling. 


\section{ACKNOWLEDGMENTS}

This work was supported by CSIRO Mineral Resources, a CSIRO Cutting Edge Science Symposium Grant and the University of Queensland's Sustainable Minerals Institute who co-organized the workshop "Valuation of Social Licence under Uncertainty" (June 2015, Brisbane). We are grateful to all participants at the Cutting Edge Science Workshop for their comments and suggestions. Our special thanks go to Dr Kieren Moffat, Dr Ross Sparks, Dr Glen Corder, and Professor David Brereton for many fruitful discussions.

\section{REFERENCES}

Boutilier, R.G., and Thomson, I. (2011). Modeling and measuring the Social Licence to operate: fruits of a dialog between theory and practice. International Mine Management, Queensland, Australia.

Brereton, D. (2015). Growing importance of social licence to operate in the minerals industry. A keynote presentation at the Cutting Edge Science Symposium, Brisbane, June 2015.

Charles, C. (2015). Social licence: an industry perspective. A keynote presentation at the Cutting Edge Science Symposium, Brisbane, June 2015.

Cooper, S. (2014). The financial value of social licence. Life of Mine Conference, July 2014.

Davis, R., and Frank, D.M. (2011). The costs of conflict with local communities in the extractive industry, SR Mining, Chile.

Dorobantu S, Henisz W and Nartey (2013) Stakeholder capital and performance in tough times, Working paper, The Wharton School, University of Pennsylvania.

IFC (2012), Financial Valuation Tool for Sustainability Investments: User Guide, IFC, MIGA, Deloitte, January 2012.

Franks, D.M., Davis, R., Bebbington, A.J., Ali, S.H., Kemp, D., and Scurrah, M. (2014). Conflict translates environmental and social risk into business costs. PNAS, 111, 7576-7581.

Henisz, W.J., Dorobantu, S., and Nartey, L.J. (2013). Spinning gold: the financial returns to stakeholder engagement, Strategic management Journal, 10.

Henisz, W. (2015). Valuing, building and maintaining cooperation with stakeholder: insight from media content coding. A keynote presentation at the Cutting Edge Science Symposium, Brisbane, June 2015.

ICMM (2015), Research on company-community conflict, Social and Economic Development, March 2015.

Jones, V.N., Lukic, J., Bhalla, A., and Tapiero, D. (2011). Measuring Returns on Community Investments. First International Conference on Social Responsibility in Mining (SR Mining2011), Santiago, Chile.

Kahneman, D. and Tversky, A. (1979). Prospect theory: an analysis of decisions under risk. Econometrica, 47(2), 263-291.

Lacey, J., Parson, R., and Moffat, K. (2012). Exploring the concept of a Social Licence to Operate in the Australian minerals industry, CSIRO, MDU.

Lacey, J., Moffat, K., Zhang, A., and Ashworth, P. (2014). Earning a social licence for alternative transport fuels, in Transport Fuels from Australia's Gas Resources, 207-218.

Moffat, K., and Zhang, A. (2014). The path to social licence to operate: an integrative model explaining community acceptance of mining. Resources Policy, 39, 61-7.

Moffat, K. (2015). The path to social licence to operate, A keynote presentation at the Cutting Edge Science Symposium, Brisbane, June 2015.

Prno, J., and Slocombe, D.S. (2014). A system-based conceptual framework for assessing the determinants of a social licence to operate in the mining industry. Environmental Management, 53, 672-689.

Sparks, R. (2015). Examples of modelling and monitoring social value or behavior. A keynote presentation at the Cutting Edge Science Symposium, Brisbane, June 2015.

Thomson, I., and Boutilier, R.G. (2011). Social licence to operate. In P.Darling (Ed.), SME mining engineering handbook, 673-690.

Zhang, A., and Moffat, K. (2015). The path to social licence to operate: an integrative model explaining community acceptance of mining. Resources Policy, 44, 25-34. 